\title{
Beyond the diffraction limit of optical/IR interferometers ${ }^{\star} \star \star \star$ II. Stellar parameters of rotating stars from differential phases
}

\author{
M. Hadjara ${ }^{1,2}$, A. Domiciano de Souza $^{1}$, F. Vakili ${ }^{1}$, S. Jankov ${ }^{3}$, F. Millour ${ }^{1}$, A. Meilland ${ }^{1}$, Z. Khorrami ${ }^{1}$, A. Chelli $^{4}$, \\ C. Baffa ${ }^{5}$, K.-H. Hofmann ${ }^{6}$, S. Lagarde ${ }^{1}$, and S. Robbe-Dubois ${ }^{1}$
}

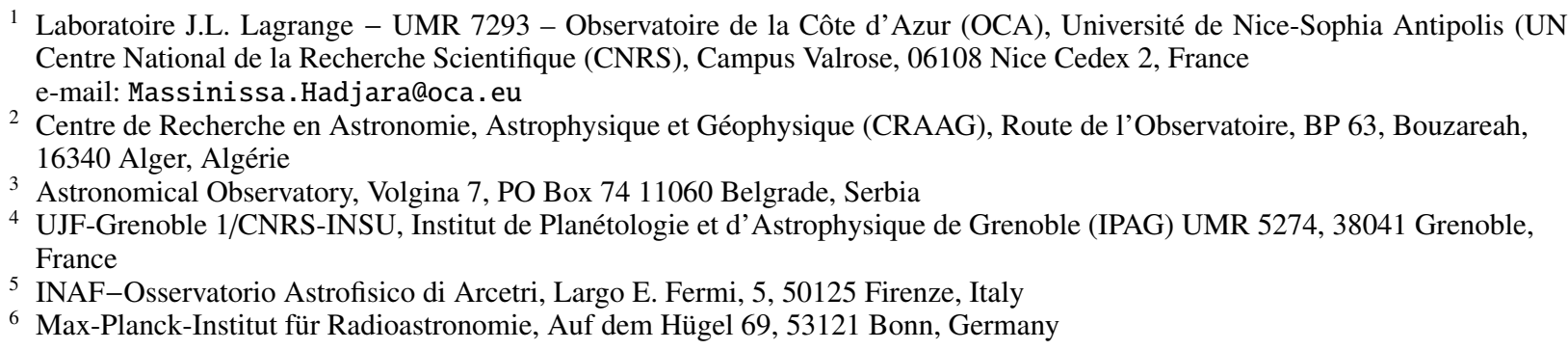

Received 12 May 2014 / Accepted 4 July 2014

\begin{abstract}
Context. As previously demonstrated on Achernar, one can derive the angular radius, rotational velocity, axis tilt, and orientation of a fast-rotating star from the differential phases obtained by spectrally resolved long baseline interferometry using earth-rotation synthesis.

Aims. We applied this method on a small sample of stars for different spectral types and classes, in order to generalize the technique to other rotating stars across the H-R diagram and determine their fundamental parameters.

Methods. We used differential phase data from the AMBER/VLTI instrument obtained prior to refurbishing its spectrometer in 2010. With the exception of Fomalhaut, which has been observed in the medium-resolution mode of AMBER $(\lambda / \delta \lambda \approx 1500)$, our three other targets, Achernar, Altair, and $\delta$ Aquilae offered high-resolution $(\lambda / \delta \lambda \approx 12000)$ spectro-interferometric data around the $\mathrm{Br} \gamma$ absorption line in $K$ band. These data were used to constrain the input parameters of an analytical, still realistic model to interpret the observations with a systematic approach for the error budget analysis in order to robustly conclude on the physics of our 4 targets. We applied the super resolution provided by differential phases $\phi_{\text {diff }}$ to measure the size (equatorial radius $R_{\text {eq }}$ and angular diameter $\emptyset_{\text {eq }}$ ), the equatorial rotation velocity $\left(V_{\mathrm{eq}}\right)$, the inclination angle $(i)$, and the rotation axis position angle $\left(\mathrm{PA}_{\text {rot }}\right)$ of 4 fast-rotating stars: Achernar, Altair, $\delta$ Aquilae, and Fomalhaut. The stellar parameters of the targets were constrained using a semi-analytical algorithm dedicated to fast rotators SCIROCCO.

Results. The derived parameters for each star were $R_{\mathrm{eq}}=11.2 \pm 0.5 R_{\odot}, V_{\mathrm{eq}} \sin i=290 \pm 17 \mathrm{~km} \mathrm{~s}^{-1}, \mathrm{PA}_{\mathrm{rot}}=35.4^{\circ} \pm 1.4^{\circ}$, for Achernar; $R_{\mathrm{eq}}=2.0 \pm 0.2 R_{\odot}, V_{\mathrm{eq}} \sin i=226 \pm 34 \mathrm{~km} \mathrm{~s}^{-1}, \mathrm{PA}_{\mathrm{rot}}=-65.5^{\circ} \pm 5.5^{\circ}$, for Altair; $R_{\mathrm{eq}}=2.2 \pm 0.3 R_{\odot}, V_{\mathrm{eq}} \sin i=74 \pm 35 \mathrm{~km} \mathrm{~s}{ }^{-1}$, $\mathrm{PA}_{\mathrm{rot}}=-101.2^{\circ} \pm 14^{\circ}$, for $\delta$ Aquilae; and $R_{\mathrm{eq}}=1.8 \pm 0.2 R_{\odot}, V_{\mathrm{eq}} \sin i=93 \pm 16 \mathrm{~km} \mathrm{~s}^{-1}, \mathrm{PA}_{\mathrm{rot}}=65.6^{\circ} \pm 5^{\circ}$, for Fomalhaut. They were found to be compatible with previously published values from differential phase and visibility measurements, while we were able to determine, for the first time, the inclination angle $i$ of Fomalhaut $\left(i=90^{\circ} \pm 9^{\circ}\right)$ and $\delta$ Aquilae $\left(i=81^{\circ} \pm 13^{\circ}\right)$, and the rotation-axis position angle $\mathrm{PA}_{\text {rot }}$ of $\delta$ Aquilae.

Conclusions. Beyond the theoretical diffraction limit of an interferometer (ratio of the wavelength to the baseline), spatial super resolution is well suited to systematically estimating the angular diameters of rotating stars and their fundamental parameters with a few sets of baselines and the Earth-rotation synthesis provided a high enough spectral resolution.
\end{abstract}

Key words. stars: rotation - stars: general - methods: observational - methods: numerical - techniques: interferometric techniques: high angular resolution

\section{Introduction}

The rotation of stars can reach more than $80 \%$ of their critical or breakup velocity $v_{\mathrm{c}}=\sqrt{\left(G M / R_{\mathrm{c}}\right)}$ in some specific cases. Here, $G$ is the gravitational constant, $M$ the mass of the star, and $R_{\mathrm{c}}$ the equatorial radius at this velocity. The Eddington factor can

* Based on observations performed at the European Southern Observatory, Chile, under ESO AMBER-consortium GTO program IDs 084.D-0456 081.D-0293 and 082.C-0376.

$\star \star$ Figure 5 is available in electronic form at http://www. aanda.org be ignored in the case of the stars studied in this paper presenting low luminosity. Fast-rotating stars exhibit a number of peculiar characteristics, such as geometrical flattening, coupled with gravitational darkening (von Zeipel 1924), making the poles apparently hotter than the equator. Gravity darkening has a profound impact on the physics of the star, with important observational consequences. For example, the models from Collins \& Sonneborn (1977) indicate a two-component spectral energy distribution (SED) for these stars, with an infrared (IR) excess due to gravity darkening. It is therefore not easy to place these stars in one single spectral classification, since the observed 
SED depends on their rotational velocity and inclination angle (Maeder \& Peytremann 1972). In addition, rotation-induced mechanisms, such as meridional circulation or turbulence, may affect the internal structure of the star and its evolution (Meynet 2009). Thus, fast rotators can be considered as a physics laboratory for studying the impact of rotation on the stellar structure and evolution.

Optical-long baseline interferometry (OLBI) is a key observing technique for directly constraining the stellar diameter and the so-called von Zeipel effect (von Zeipel 1924; Domiciano de Souza et al. 2003; McAlister et al. 2005; Aufdenberg et al. 2006; Peterson et al. 2006; Monnier et al. 2007; Zhao et al. 2009; Che et al. 2011; van Belle 2012; Delaa et al. 2013). The study of rotation across the H-R diagram may also give information on the internal structure of stars of different spectral types and luminosity classes and the physics of their atmosphere (Espinosa Lara \& Rieutord 2011).

OLBI is classically based on measuring visibility amplitudes at different baselines to determine the angular size of the target being observed. However, when a rotating star is studied with spectrally resolved interferometry, the Doppler shift across a line, which implicitly contains spatial information, together with the change in the photocenter relative to this shift (Lagarde 1994), can measure the angular size of the visible hemisphere of the star.

In addition, spectrally resolved long-baseline interferometry - also called differential interferometry (DI; Petrov 1989; Lachaume 2003) once combined with Earth-rotation synthesis and/or the use of simultaneous baselines from three or more telescopes, offers new possibilities for observing the details of rotationally flattened stars and directly checking whether the von Zeipel effect applies to different spectral types and luminosity classes (van Belle 2012). The application of DI in practice demands, however, special data analysis to access the so-called differential phase (Vakili et al. 1998), which in turn needs appropriate calibration procedures that go beyond the usual traditional angular diameter estimates versus visibility curves extrema and/or zeros.

This paper scrutinizes four stars of different spectral types, namely:

- Achernar ( $\alpha$ Eridani, B6Vep spectral type),

- Altair ( $\alpha$ Aquilae, A7V spectral type),

- $\delta$ Aquilae (HD182640, F0IV spectral type)

- Fomalhaut ( $\alpha$ Piscis Austrini, A4V spectral type).

These stars were observed using DI with the AMBER focal instrument of the VLTI (Petrov et al. 2007). This comparative study focuses on the rotational characteristics of these stars. To interpret these data, we developed a dedicated analytical model called SCIROCCO (Simulation Code of Interferometricobservations for ROtators and CirCumstellar Objects, Hadjara et al. 2012, 2013) in order to derive the fundamental and geometrical parameters of our sample of stars.

The present paper is organized as follows: we first introduce the stars, their observations, and data reduction (Sect. 2). We recall the principles of differential phase measurements and present the SCIROCCO model (Sect. 3). In Sect. 4, we validate the SCIROCCO model, comparing it with previously reported measures of Achernar. We then determine the parameters of Altair, $\delta$ Aquilae, and Fomalhaut in Sect. 5. These results, together with future applications of SCIROCCO, are discussed for a broader study of fast-rotating stars with AMBER/VLTI interferometry across the HR diagram (Sect. 6).

\section{Observations and data reduction}

\subsection{The sample stars}

The data of the four studied stars in this paper are available from ESO archive. Achernar ( $\alpha$ Eri, HR 472, HD 10144) is a rotationnally flattened $\mathrm{Be}$ star with an oblateness ratio reported between 1.41 and 1.56 typically with $3 \%$ accuracy (Domiciano de Souza et al. 2003). Subsequent studies (Kervella \& Domiciano de Souza 2006) considered a polar mass-loss component added to the photospheric disk, for a star with rotational parameter $V_{\mathrm{eq}} \sin i=225 \mathrm{~km} \mathrm{~s}^{-1}$ (from previous spectroscopic estimates by Slettebak 1982). Then a small residual disk (Carciofi et al. 2008) and/or the presence of a companion by Kervella et al. (2008) were invoked to explain the apparent strong flattening for Achernar from interferometric assymetric data. However, Kanaan et al. (2008) considered the circumstellar environment (CSE) contribution as important for explaining the strong flatness. Overall, Domiciano de Souza et al. (2012, Paper I), gave the recent parameters of Achernar as for its equatorial radius to be $11.6 R_{\odot}$, its equatorial velocity $298 \mathrm{~km} \mathrm{~s}^{-1}$, the inclination $101^{\circ}$ and the position angle $34.7^{\circ}$.

Altair ( $\alpha$ Aql, HR 7557, HD 187642) is a bright $(V=0.77)$ A7IV-V star (Johnson \& Morgan 1953) that is known to be a rapid rotator $\left(240 \mathrm{~km} \mathrm{~s}^{-1}\right.$ from Slettebak 1955). These characteristics, together with its location (close to celestial equator, therefore observable in both the northern and southern hemispheres), make Altair a prime target among the fast rotators. For example, Buzasi et al. (2005) show that it is a variable of the $\delta$ Scuti type by detecting several oscillating frequencies in this star. Several interferometric and spectroscopic observations indicate a $V_{\text {eq }} \sin i$ value between $190 \mathrm{~km} \mathrm{~s}^{-1}$ and $250 \mathrm{~km} \mathrm{~s}^{-1}$ (Abt \& Morrell 1995; van Belle et al. 2001; Royer et al. 2002; Reiners \& Royer 2004b, among others). Domiciano de Souza et al. (2005) used the interferometer VINCI/VLTI and a model for fast rotators, including Roche approximation, limb-darkening, and von Zeipel gravity-darkening, to find the same value of $V_{\mathrm{eq}} \sin i=227 \mathrm{~km} \mathrm{~s}^{-1}$ as in Reiners \& Royer (2004a), who used spectroscopy for determine the constraints on Altairs inclination angle and differential rotation from the global rotational broadening profile derived from about 650 spectral absorption lines. Most recently, Monnier et al. (2007) have reconstructed an image of the surface of Altair from CHARA observations, inferring several fundamental parameters: inclination, position angle, effective temperature, and radii of the pole and of the equator. They derived the values of $V_{\mathrm{eq}} \sin i=240 \mathrm{~km} \mathrm{~s}^{-1}$ and $241 \mathrm{~km} \mathrm{~s}^{-1}$.

The source $\delta$ Aquilae A ( $\delta$ Aql, HR 7377, HD 182640) is a subgiant F0IV star (Cowley \& Fraquelli 1974). Using a model atmosphere analysis of spectroscopic binaries and multiple star systems, Fuhrmann (2008) determined that the mass of this star is $1.65 M_{\odot}$, and its radius is $2.04 R_{\odot}$. Looking for signatures of differential rotation in line profiles, Reiners (2006) found an effective temperature of $7016 \mathrm{~K}$. The star $\delta$ Aquilae A presents non-radial pulsations and is a Delta Scuti variable that in addition is spinning rapidly with a $V_{\text {eq }} \sin i$ estimated around $89 \mathrm{~km} \mathrm{~s}^{-1}$ (Mantegazza \& Poretti 2005).

Fomalhaut ( $\alpha$ PsA, HR 8728, HD 216956) is a young A4V star. As the nineteenth brightest star in the sky, it is one of the closest main sequence star surrounded by a spatially resolved debris disk with an inclined ring of about $140 \mathrm{AU}$ in radius (Holland et al. 2003; Stapelfeldt et al. 2004). With HST/ACS imaging, under the assumption that the disk is intrinsically circular, Kalas et al. (2005) estimated the disk inclination 
M. Hadjara et al.: Beyond the diffraction limit of optical/IR interferometers. II.
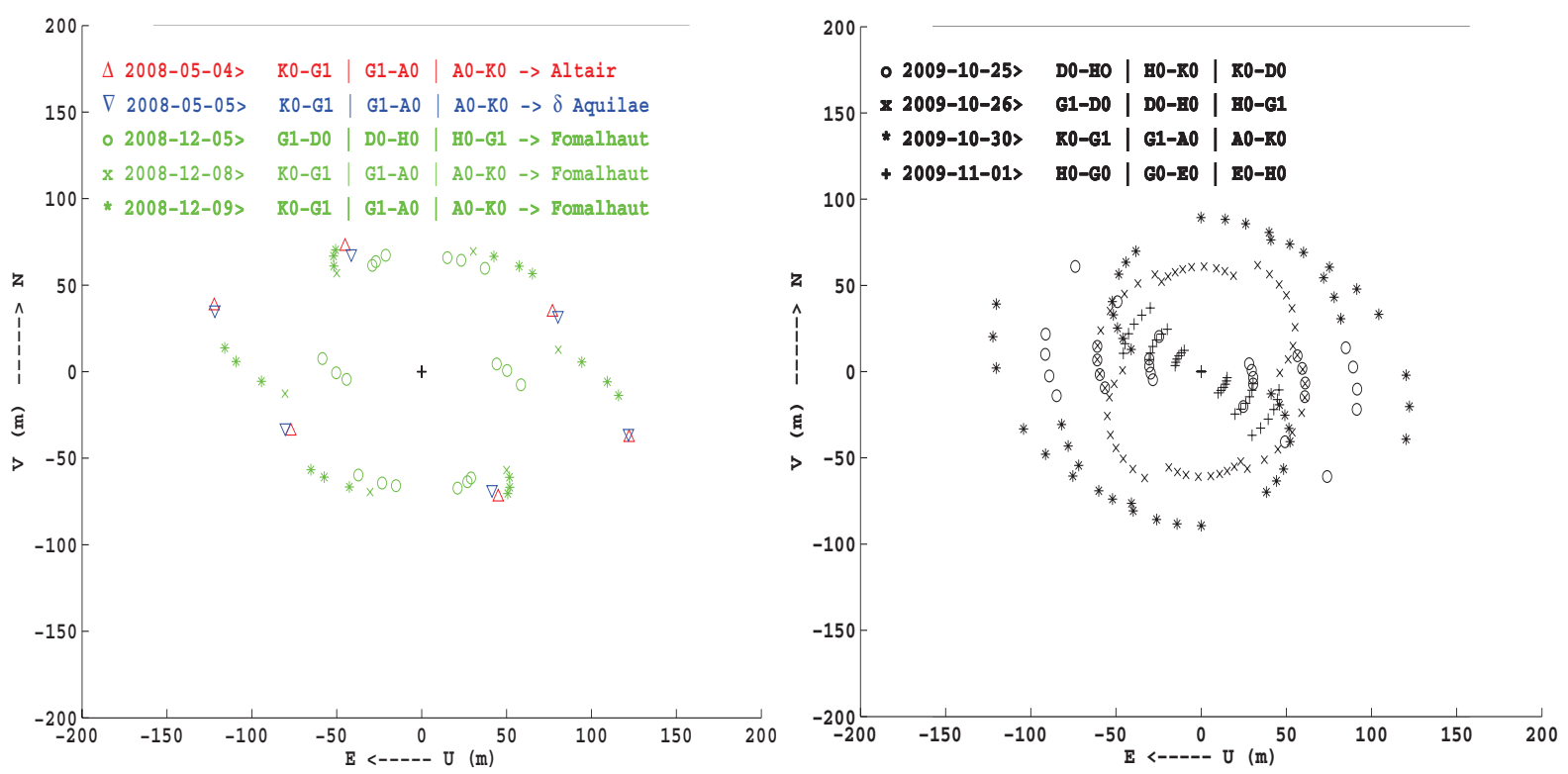

Fig. 1. Left: baselines and the corresponding $(u, v)$ coverage of VLTI/AMBER observations of Altair, $\delta$ Aquilae, and Fomalhaut. Earth-rotation synthesis spanned over $\sim 1.5 \mathrm{~h} /$ night for $\delta$ Aquilae and Altair and $\sim 5 \mathrm{~h} /$ night for Fomalhaut. Right: same data for Achernar, where Earth-rotation synthesis spanned over $\sim 5 \mathrm{~h} /$ night. Notice the rather dense sampling of the Fourier space for the target stars.

$i_{\text {disk }}=65^{\circ} .9 \pm 0.4^{\circ}$ and the position angle of $\mathrm{PA}_{\text {disk }}=156^{\circ} \pm 0.3^{\circ}$. Kalas et al. (2008) confirm the presence of a planetary companion. Royer et al. (2007) estimated the equatorial velocity $V_{\text {eq }}=93 \mathrm{~km} \mathrm{~s}^{-1}$ and Di Folco et al. (2004) find the equatorial radius $R_{\text {eq }}=1.1$ mas $\left(1.8 R_{\odot}\right)$. Recently, Le Bouquin et al. (2009) used the spectrally resolved near-IR long baseline interferometry to obtain spectro-astrometric measurements across the $\mathrm{Br} \gamma$ absorption line, to find a position angle $\mathrm{PA}_{\text {star }}=65^{\circ} \pm 0.3^{\circ}$ for the stellar rotation axis, perpendicular to the literature measurement for the disk position angle $\left(\mathrm{PA}_{\text {disk }}=156^{\circ} .0 \pm 0.3^{\circ}\right)$, and they suggest backward-scattering properties for the circumstellar dust grains. However, they did not infer the star's radius from their measurements, which we do in this article with the same dataset.

\subsection{The observations}

All the above stars were observed with the AMBER/VLTI instrument located at Cerro Paranal, Chile, with the Auxiliary Telescopes (ATs). They were observed using the high spectral resolution mode of $\operatorname{AMBER}(\lambda / \Delta \lambda \approx 12000)$ with the exception of Fomalhaut, observed using the medium spectral resolution mode $(\lambda / \Delta \lambda \approx 1500)$. Table 1 provides the observation log of Altair, $\delta$ Aquilae, and Fomalhaut. The UV coverage for all studied stars is provided in Fig. 1. The Achernar's observations (that are not included in Table 1) are the same as in Paper I.

\subsection{Instrumental biais trouble-shooting and data calibration}

The differential phase $\phi_{\text {diff }}$ obtained from AMBER data reduction algorithm is related to the object's Fourier phase $\phi_{\text {obj }}$ by (e.g., Millour et al. 2011, 2006):

$\phi_{\mathrm{diff}}(u, v)=\phi_{\mathrm{obj}}(u, v)-a(u, v)-b(u, v) / \lambda$,

where the spatial frequency coordinates $u$ and $v$ depend on the wavelength $\lambda$, the projected baseline length $B_{\text {proj }}$ and the baseline position angle PA (from north to east; $u=B_{\text {proj }} \sin (\mathrm{PA}) / \lambda$ and
Table 1. VLTI/AMBER observations of Altair, $\delta$ Aquilae, and Fomalhaut with details on the dates, times, and baseline triplets.

\begin{tabular}{|c|c|c|c|}
\hline Object & Date and time & $\begin{array}{c}\text { Baseline length } \\
B_{\text {proj }}(\mathrm{m})\end{array}$ & $\begin{array}{c}\text { Baseline PA } \\
\mathrm{PA}\left({ }^{\circ}\right)\end{array}$ \\
\hline \multicolumn{4}{|c|}{ Altair $\longrightarrow$ Observation with AT Triplet K0-G1-A0: } \\
\hline Altair & 2008-05-04T09:50 & $80,87,127$ & $-148,-68,-106$ \\
\hline hd184406 & 2008-05-04T10:28 & $85,79,127$ & $-145,-67,-108$ \\
\hline \multicolumn{4}{|c|}{$\delta$ Aquilae $\longrightarrow$ Observation with AT Triplet K0-G1-A0: } \\
\hline hd184406 & 2008-05-05T09:08 & $79,89,125$ & -150 \\
\hline$\delta$ Aquilae & 2008-05-05T09:49 & $85,84,128$ & $-148,-66,-107$ \\
\hline \multicolumn{4}{|c|}{ Fomalhaut $\longrightarrow$ Observation with AT Triplet G1-D0-H0: } \\
\hline Fomalhaut & 2008-12-05T00:20 & $70,59,71$ & $-32,83,18$ \\
\hline $88 \mathrm{Aqr}$ & 2008-12-05T01:13 & $66,55,71$ & $-26,82,20$ \\
\hline Fomalhaut & 2008-12-05T01:30 & $68,50,69$ & $-20,91,23$ \\
\hline$\gamma \mathrm{Scl}$ & 2008-12-05T01:48 & $69,51,68$ & $-20,93,23$ \\
\hline Fomalhaut & 2008-12-05T02:06 & $68,44,68$ & $-13,96,25$ \\
\hline $88 \mathrm{Aqr}$ & 2008-12-05T02:24 & $63,43,70$ & $-13,87,25$ \\
\hline \multicolumn{4}{|c|}{ Fomalhaut $\longrightarrow$ Observation with AT Triplet K0-G1-A0: } \\
\hline Fomalhaut & 2008-12-08T02:15 & $76,76,81$ & $-138,-24,-81$ \\
\hline 88Aqr & 2008-12-08T02:31 & $80,68,79$ & $-141,-25,-91$ \\
\hline Fomalhaut & 2008-12-09T00:10 & $87,86,117$ & $-144,-49,-97$ \\
\hline $88 \mathrm{Aqr}$ & 2008-12-09T00:27 & $89,83,117$ & $-145,-51,-100$ \\
\hline Fomalhaut & 2008-12-09T00:40 & $85,84,110$ & $-142,-43,-93$ \\
\hline $88 \mathrm{Aqr}$ & 2008-12-09T00:58 & $87,78,107$ & $-143,-44,-97$ \\
\hline Fomalhaut & 2008-12-09T01:31 & $80,79,94$ & $-139,-33,-87$ \\
\hline$\gamma \mathrm{Scl}$ & 2008-12-09T01:51 & $79,81,95$ & $-138,-32,-84$ \\
\hline
\end{tabular}

Notes. The calibration stars are hd184406, 88Aqr, and $\gamma \mathrm{Scl}$.

$\left.v=B_{\text {proj }} \cos (\mathrm{PA}) / \lambda\right)$. The parameters $a$ and $b$ correspond to an offset and a slope, given in appropriate units.

Data have been reduced using version 3.1 of the amdlib software (Chelli et al. 2009; Tatulli et al. 2007). We adopted a standard frame selection based on fringe signal-to-noise ratio (Millour et al. 2007) and kept the 50\% best frames calibrated later using appropriate software by Millour et al. (2008). The visibility amplitudes could not be reliably calibrated due to too chaotic a transfer function. Indeed, FINITO did not provide the 

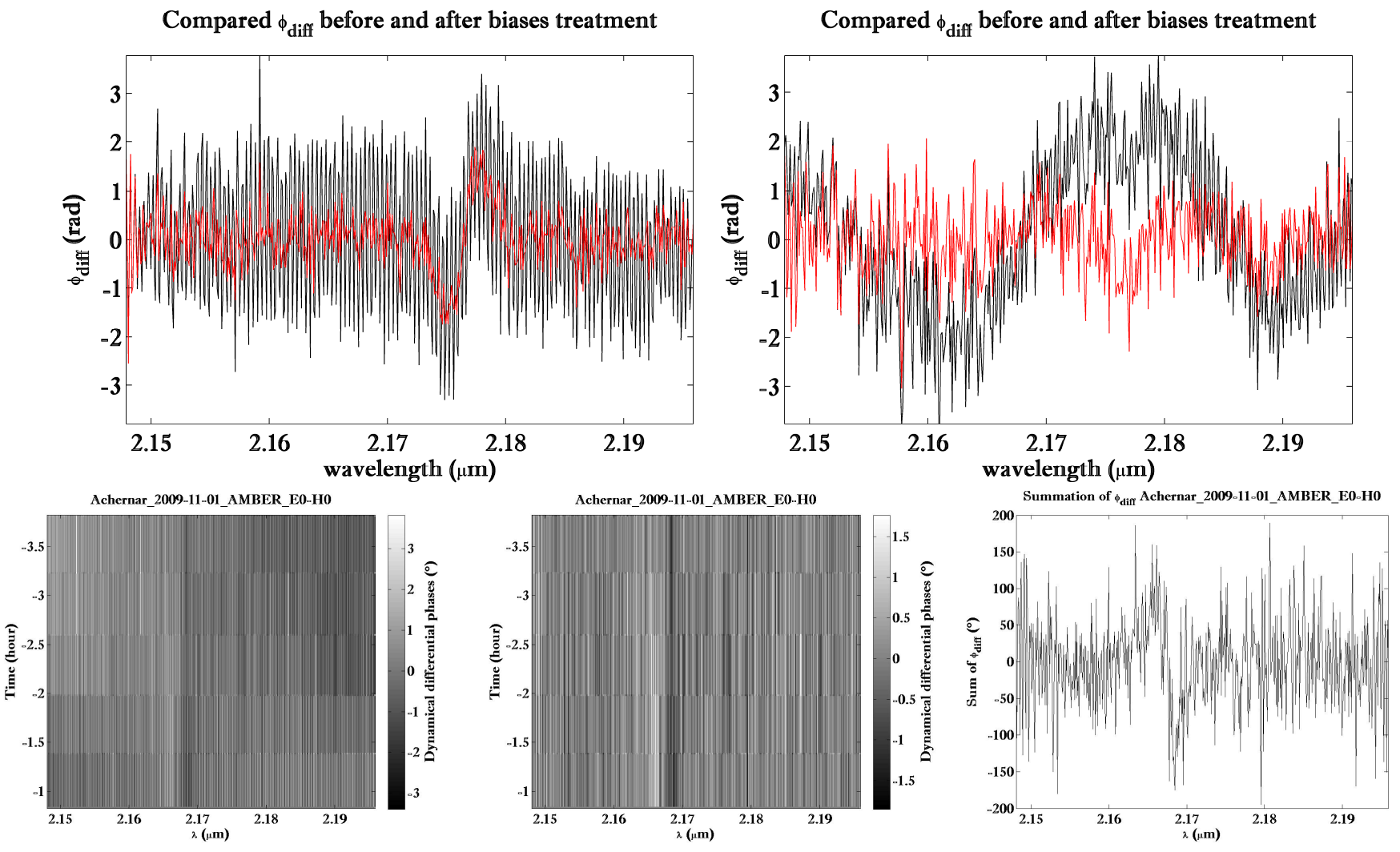

Fig. 2. Top: AMBER instrumental fringing effect on the differential phases as a function of wavelength. Left: Achernar's differential phases showing high-frequency beating (black line), and the same filtered out (red line). Right: same as for Altair. Bottom: left: Achernar's dynamical differential phases before removing the fringing effect, for the observing night of 2009 Nov. 01, for the E0-H0 baseline. Center: after bias removal, the rotation effect around the Brackett $\gamma$ line becomes more visible as a dark vertical strip next to a bright vertical strip. Right: by projecting the differential phases as a function of wavelength, one gets the typical s-shaped effect as a function of wavelength.

additional data necessary at that time to properly calibrate the absolute visibilities. Our final dataset include the source spectrum, differential visibilities, differential phases, and closure phases.

Owing to the defective IRIS-feeding dichroic plate in the VLTI optical train in front of AMBER, a high-frequency beating affected all our measurements (spectrum, visibilities, phases) and was also present in the calibrated data (Fig. 2). Owing to the polarizing prisms of AMBER, a low-frequency beating (AMBER "socks") was also present in our data. The highfrequency beating appears at a specific frequency in the wavenumber domain. We removed it by Fourier filtering at that specific frequency. The second beating was corrected by subtracting a sine wave fit to the continuum signal.

We performed a wavelength calibration by using the positions of telluric lines. We normalized the spectrum by adjusting and dividing a fourth order polynomial to the continuum. Figure 2 (top) shows an comparative example of the AMBER differential phases before and after processing, while Fig. 2 (bottom) shows dynamical differential phases before and after removing of the instrumental biases.

Our final data set consists of:

- Altair: $6(=2 \times 3$ baselines $) \phi_{\text {diff }}(\lambda)$ curves centered on $\operatorname{Br} \gamma$, - $\delta$ Aquilae: $6(=2 \times 3$ baselines $) \phi_{\text {diff }}(\lambda)$ curves centered on $\operatorname{Br} \gamma$,

- Fomalhaut: $18(=6 \times 3$ baselines $) \phi_{\text {diff }}(\lambda)$ curves centered on Br $\gamma$,

- Achernar: the same $84(=28 \times 3$ baselines $) \phi_{\text {diff }}(\lambda)$ curves as in Paper I, used here for reference to our analytical model.
The high spectral resolution mode of $\operatorname{AMBER}(\lambda / \Delta \lambda=12000)$ leads to a velocity resolution of $\simeq 25 \mathrm{~km} \mathrm{~s}^{-1}$, while the mediumresolution mode $(\lambda / \Delta \lambda=1500)$ leads to the velocity resolution of $\simeq 200 \mathrm{~km} \mathrm{~s}^{-1}$. Projected equatorial rotational velocities $V_{\text {eq }} \sin i$ above $\sim 150 \mathrm{~km} \mathrm{~s}^{-1}$ would ensure that inside the $\mathrm{Br} \gamma$ line, the attained resolution is $\sim 12$ individual spectral canals in high resolution and two spectral canals in the middle resolution observational mode. In such cases, rotation effects need to be accounted for when modeling phase signatures in order to be consistent with the physics of the studied star.

\section{SCIROCCO code for modeling rotating stars}

To interpret these spectro-interferometric observations, we developed a chromatic semi-analytical model for fast rotators: Simulation Code of Interferometric-observations for ROtators and CirCumstellar Objects (SCIROCCO). The code, implemented in Matlab ${ }^{1}$, allows computing monochromatic intensity maps of uniformly rotating, flattened, and gravity-darkened stars from a semi-analytical approach. SCIROCCO is described below, and in the next section it is used to investigate the dependence of observed interferometric differential phases of the four stars on the relevant input physical parameters.

1 MATrix LABoratory. 
M. Hadjara et al.: Beyond the diffraction limit of optical/IR interferometers. II.
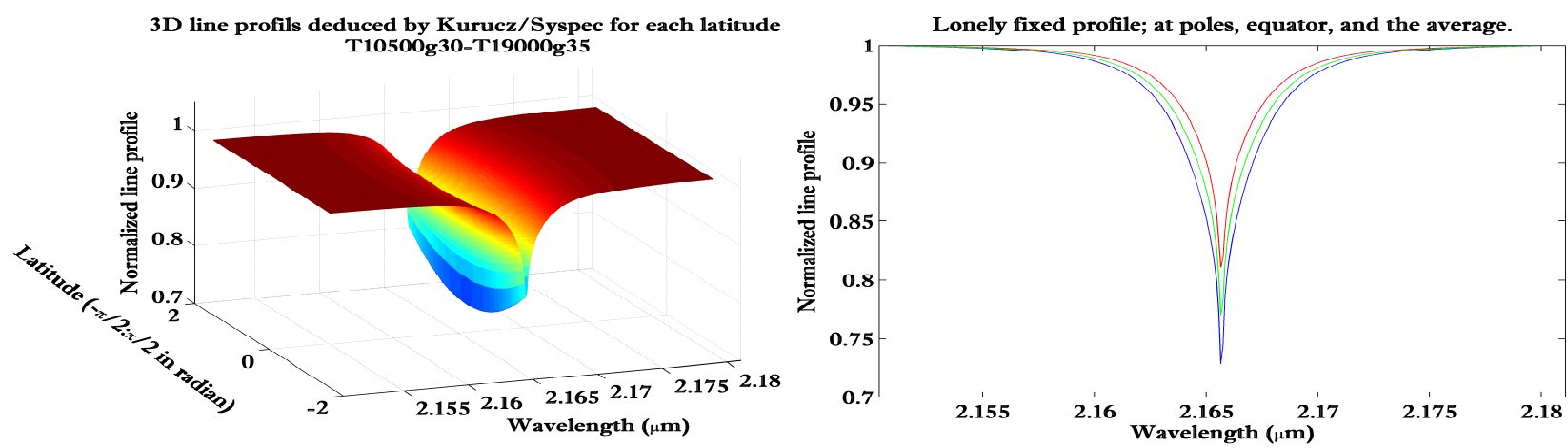

Fig. 3. Left: 3D Brackett $\gamma$ local line profile representation for a star with $\left[\bar{T}_{\text {eff }}, \log g\right]_{\text {pole }}=\left[10500 \mathrm{~K}, 30 \mathrm{~cm} / \mathrm{s}^{2}\right]$ at the poles and $\left[\bar{T}_{\text {eff }}, \log g\right]_{\mathrm{eq}}=$ $\left[19000 \mathrm{~K}, 35 \mathrm{~cm} / \mathrm{s}^{2}\right]$ at the equator from Kurucz/Synspec model. The polar line profiles (in the board) have less amplitude than the equatorial one in the middle. Right: 3 panels of 1D cuts of the same 3D figure at different latitudes; at the poles (red line), at the equator (blue) and the average (in green).

\subsection{Surface shape}

The stellar surface is analytically described by an oblate ellipsoid of revolution with the semi-minor axis $R_{\mathrm{p}}$ in the direction of the rotation axis and semi-major axis given by the equatorial radius $R_{\text {eq }}$. The polar-to-equatorial radii ratio is taken from the Roche model (Domiciano de Souza et al. 2002):

$\frac{R_{\mathrm{p}}}{R_{\mathrm{eq}}}=1-\frac{V_{\mathrm{eq}}^{2} R_{\mathrm{p}}}{2 G M}=\left(1+\frac{V_{\mathrm{eq}}^{2} R_{\mathrm{eq}}}{2 G M}\right)^{-1}$

where $G$ is the gravitational constant, $V_{\text {eq }}$ the equatorial linear rotation velocity, and $M$ the mass of the star.

The minor-to-major axis ratio of the oblate spheroid projected onto the sky plane (apparent ellipse) depends on the polar inclination angle $i$ and the orientation of the ellipse, which is defined by the position angle $\mathrm{PA}_{\text {rot }}$ of the minor (rotation) axis projected onto the sky (position angle of the minor axis).

\subsection{Gravity darkening}

Following the recent spectro-interferometric works on fast rotators, we consider a von Zeipel-like (von Zeipel 1924) form for the gravity darkening effect with a $\beta$ coefficient:

$T_{\mathrm{eff}}(\theta)=\left(\frac{C}{\sigma}\right)^{0.25} g_{\mathrm{eff}}^{\beta}(\theta)$

where $T_{\text {eff }}(\theta)$ and $g_{\text {eff }}(\theta)$ are the effective surface temperature and gravity, which depend on the co-latitude $\theta ; \beta$ is the gravity darkening coefficient; and $\sigma$ is the Stefan-Boltzmann constant. The constant $C$ is given by

$C=\frac{\sigma \bar{T}_{\text {eff }}^{4} S_{\star}}{\int g_{\text {eff }}^{4 \beta}(\theta) \mathrm{d} S}$

where $\bar{T}_{\text {eff }}$ is the average effective temperature over the stellar surface (ellipsoidal surface in our model) and $S_{\star}$ the stellar surface. We adopt the Roche model expression for the effective gravity $g_{\text {eff }}(\theta)$, but considering the equation of the oblate ellipsoid of revolution for the dependence of the radius with colatitude $\theta$. Given this definition of $g_{\mathrm{eff}}(\theta)$, the gravity darkening is totally defined by the above equations with input parameters $\bar{T}_{\text {eff }}$ and $\beta$.

\subsection{Continuum intensity map}

Once the local effective temperature $T_{\text {eff }}(\theta)$ is defined, the specific intensity maps in the continuum $I_{\mathrm{c}}(\theta, \phi)$ (continuum specific intensity at each visible pixel of the stellar surface) are given by

$\frac{I_{\mathrm{c}}(\lambda, \theta, \phi)}{I_{0}\left(\lambda, T_{\mathrm{eff}}\right)}=1-\sum_{k=1}^{4} a_{k}(\lambda)\left(1-\mu(\theta, \phi)^{\frac{K}{2}}\right)$

where $(\theta, \phi)$ are the (co-latitude, longitude) coordinates on the stellar surface, $I_{0}$ is approximated by the analytical (black body) Planck function, and $\mu(\theta, \phi)$ is the cosine of the angle between the normal to the surface at a given point and the observer's direction. Limb darkening is analytically included following the four-parameter prescription from Claret (2000a), where the limb-darkening coefficients $a_{k}$ are chosen from tabulated values for a given photometric band, local effective gravity, and temperature. In this paper we consider solar metallicity and turbulent velocity of $2 \mathrm{~km} \mathrm{~s}^{-1}$ for the limb-darkening parameters.

\subsection{Intensity map in photospheric absorption lines}

The specific intensity maps $I(\lambda, \theta, \phi)$ across photospheric absorption lines are computed as the product of the continuum specific intensity $I_{\mathrm{c}}(\lambda, \theta, \phi)$ defined above and a local normalized intensity profile $H$, which can vary over the stellar surface both because of rotational Doppler shift, and gravity darkening $I$ is thus given by

$I(\lambda, \theta, \phi)=I_{\mathrm{c}}(\lambda, \theta, \phi) H\left(\lambda+\lambda_{0} \frac{V_{\text {proj }}(\theta, \phi)}{c}, \theta, \phi\right)$

where $V_{\text {proj }}$ is the surface rotational velocity projected onto the observer's direction, $c$ is the vacuum light velocity, and $\lambda_{0}$ the central wavelength of the line profile.

The local profile $H$ over the stellar surface is interpolated from synthetic spectra calculated with the SYNSPEC code based on stellar atmosphere models from the Kurucz ATLAS9 grid (Kurucz 1970; Hubeny \& Lanz 1995), computed with the tabulated closest values $\left[T_{\text {eff }}, \log g\right]$ according to the co-latitude deducted by SCIROCCO. Figure 3 depicts examples of local profiles interpolated from the adopted spectral synthesis code.

Once the specific intensity maps on the photospheric lines are computed, the spectro-interferometric observables are directly obtained as spectra and photocenters, and by Fourier transformations, which provide visibility amplitudes, phases, and closure phases (examples are given in the bottom row of Fig. 4). 

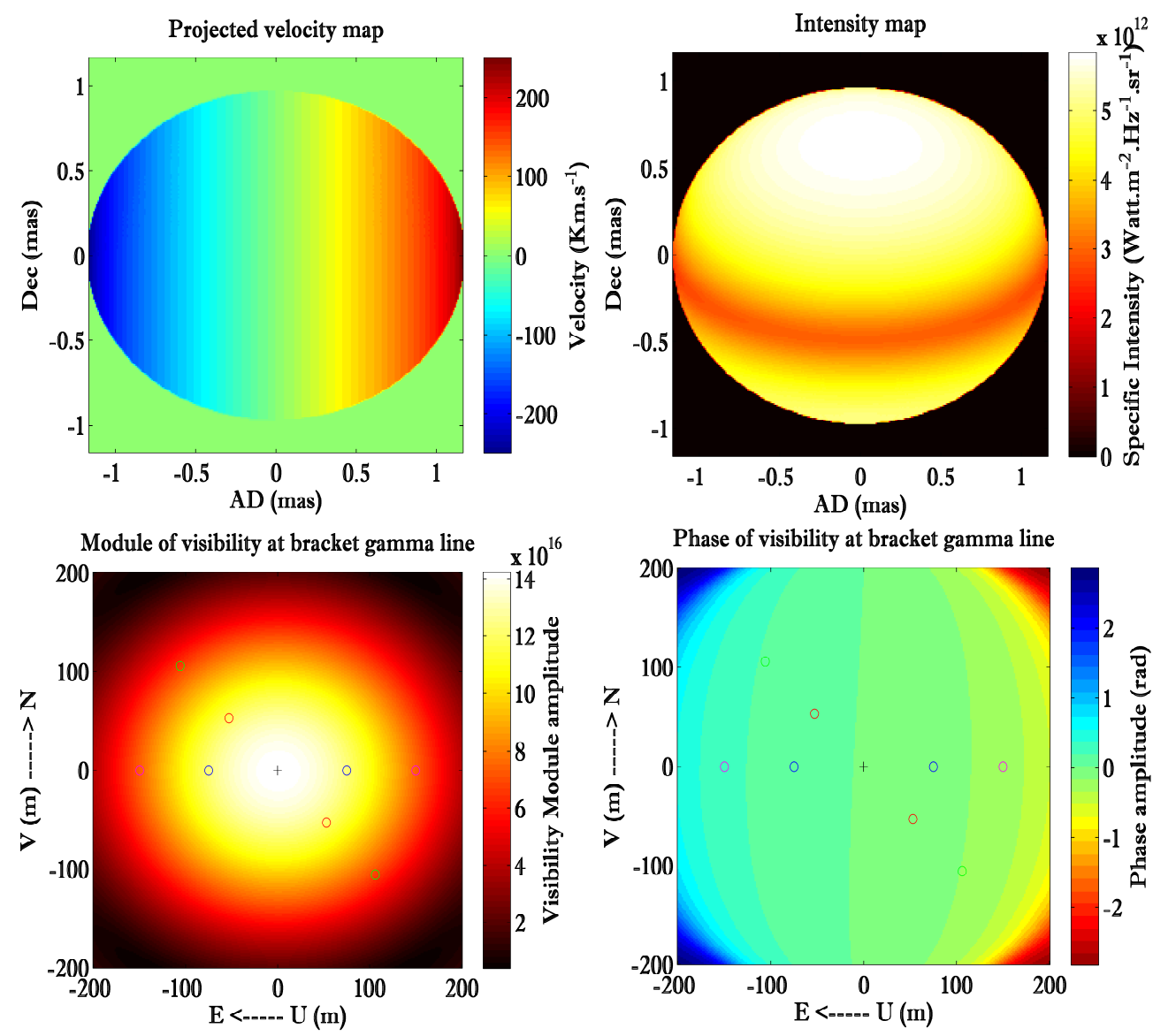

Phase of visibility at bracket gamma line
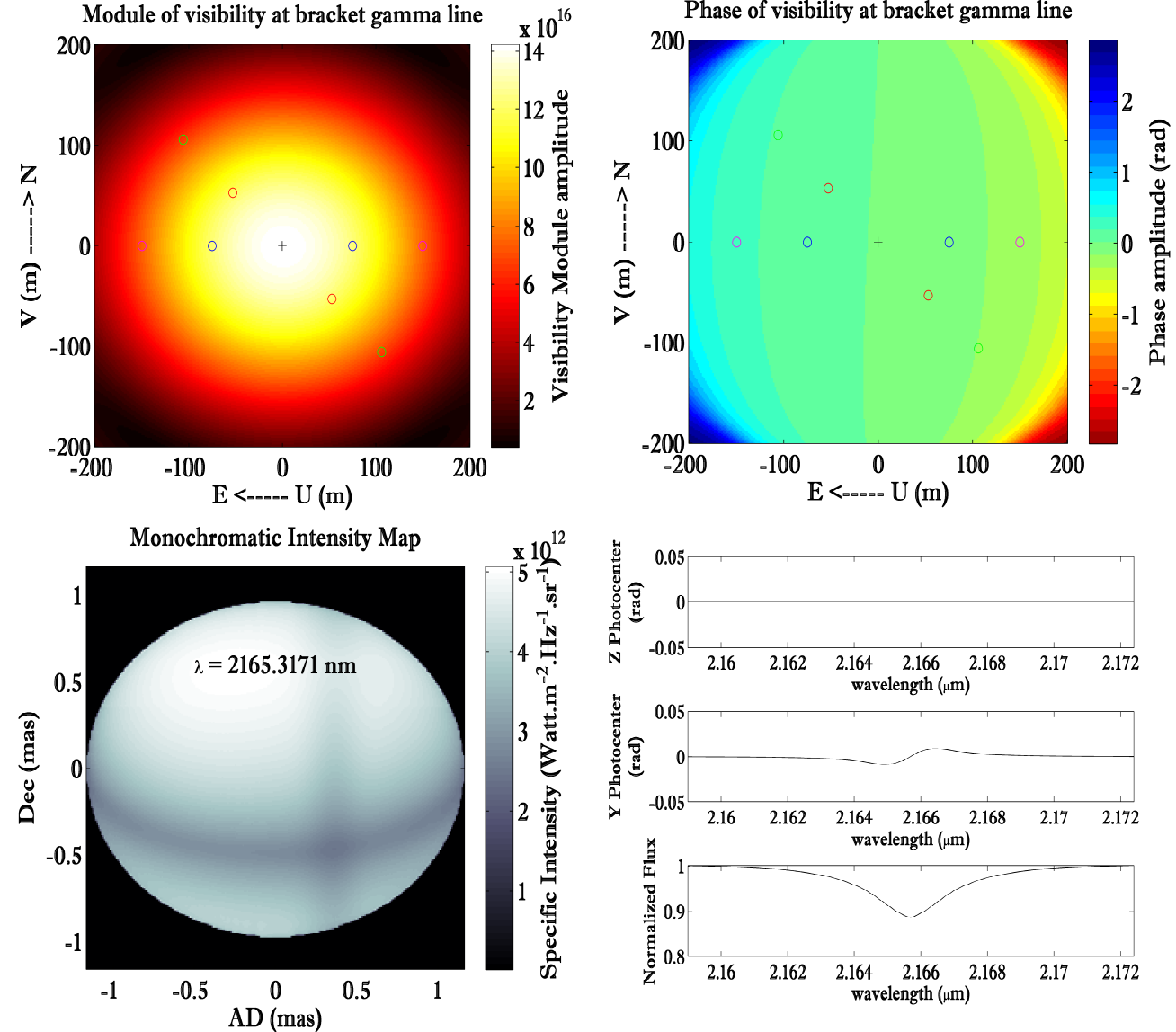

Fig. 4. Top-left: velocity map of the same model (inclination $60^{\circ}$, orientation $0^{\circ}$ ). Then for the simulated rotation a rigid body is assumed, and a velocity around $90 \%$ of the critical velocity of the star. Top-right: simulated intensity map in the continuum assuming von Zeipel gravity and limb-darkening effects where the poles are brighter than at the equator. Center-left: corresponding 2D map of the visibility modulus, where the four interferometric baselines cited above are represented by circles: ([75 $\left.\mathrm{m}, 45^{\circ}\right]$ in red circle, $\left[150 \mathrm{~m}, 45^{\circ}\right]$ in green circle, $\left[75 \mathrm{~m}, 90^{\circ}\right]$ in blue circle and $\left[150 \mathrm{~m}, 90^{\circ}\right]$ in magenta circle). Center-right: same as for the $2 \mathrm{D}$ map of the visibility phase. $\left(\left[75 \mathrm{~m}, 45^{\circ}\right]\right.$ in red circle, $\left[150 \mathrm{~m}, 45^{\circ}\right]$ in green circle, $\left[75 \mathrm{~m}, 90^{\circ}\right]$ in blue circle and $\left[150 \mathrm{~m}, 90^{\circ}\right]$ in magenta circle). Bottom-left: monochromatic intensity map for a given Doppler shift across the Brackett spectral line. Bottom-right: top: photo-center along the $Z$ axis. ( $Z$ axis represents the declination and $Y$ axis the right ascension.) Middle: photo-center along $Y$ (the photo-centers are in radians). Bottom: normalized spectrum with the Brackett $\gamma$ rotationally broadened line.

Table 2. Limb-darkening parameters adopted for Achernar.

\begin{tabular}{cc}
\hline \hline Parameters & Value \\
\hline Turbulent velocity $V_{\mathrm{T}}$ & $2 \mathrm{~km} \mathrm{~s}^{-1}$ \\
$\log g$ & 3 to $3.5 \mathrm{~cm} / \mathrm{s}^{2}$ \\
Claret $T_{\text {eff }}$ & 11500 to $19000 \mathrm{~K}$ \\
Metallicity & 0 \\
Spectral filter & $K$ \\
\hline
\end{tabular}

Thus we can give feedback on the input model parameters by comparing the synthesized observable quantities to spectrointerferometric measures within the error bars. The relevant input parameters of our model for fast-rotating stars are

- the equatorial radius $R_{\mathrm{eq}}$,

- the stellar mass $M$,

- the inclination angle $i$ and the equatorial rotation velocity $V_{\text {eq }}$ (or alternatively $V_{\mathrm{eq}} \sin i$ ), 
M. Hadjara et al.: Beyond the diffraction limit of optical/IR interferometers. II.

Table 3. Parameters and uncertainties estimated from a Levenberg-Marquardt fit of our model to the VLTI/AMBER $\phi_{\text {diff }}$ observed on studied stars, and compared with what we found in the literature.

\begin{tabular}{|c|c|}
\hline \multirow[b]{2}{*}{ Parameters } & Previously studied stars \\
\hline & Achernar \\
\hline \multirow[b]{2}{*}{ Best-fit parameter } & Comparison of best fit values those from literature \\
\hline & In the literature \\
\hline $\begin{array}{c}\text { Equatorial radius } R_{\mathrm{eq}} \\
\text { Equatorial rotation velocity } V_{\mathrm{eq}} \\
\text { Rotation-axis inclination angle } i \\
\text { Rotation-axis position angle } \mathrm{PA}_{\mathrm{rot}}\end{array}$ & $\begin{array}{c}11.2 \pm 0.5 R_{\odot} \\
295 \pm 10 \mathrm{~km} \mathrm{~s}^{-1} \\
100.0 \pm 2.5^{\circ} \\
35.4 \pm 1.4^{\circ}\end{array}$ \\
\hline$\chi^{2}$ & 2.3003 \\
\hline Fixed parameter & Value \\
\hline $\begin{array}{c}\text { Distance } d \\
\text { Mass } M \\
\text { Surface mean effective temperature } \bar{T}_{\text {eff }} \\
\text { Gravity-darkening coefficient } \beta\end{array}$ & $\begin{array}{c}44.1 \mathrm{pc}^{a} \\
6.1 \mathrm{M}_{\odot}^{a} \\
15000 \mathrm{~K}^{a} \\
0.20^{a}\end{array}$ \\
\hline Derived parameter & Value \\
\hline $\begin{array}{c}\text { Equatorial angular diameter } \emptyset_{\mathrm{eq}} \\
\text { Equatorial-to-polar radii } R_{\mathrm{eq}} / R_{\mathrm{p}} \\
\qquad V_{\mathrm{eq}} \sin i \\
V_{\mathrm{eq}} / V_{\mathrm{crit}} \\
\text { Polar effective temperature } T_{\mathrm{pol}} \\
\text { Equatorial effective temperature } T_{\mathrm{eq}} \\
\quad \text { Luminosity } \log L / L_{\odot}\end{array}$ & $\begin{array}{c}2.38 \pm 0.10 \mathrm{mas} \\
1.42 \pm 0.06 \\
290 \pm 10 \mathrm{~km} \mathrm{~s}^{-1} \\
0.94 \pm 0.03 \\
18728 \pm 327 \mathrm{~K} \\
11243 \pm 1308 \mathrm{~K} \\
3.632 \pm 0.044\end{array}$ \\
\hline
\end{tabular}

References. ${ }^{(a)}$ Domiciano de Souza et al. (2012), and references there in.

- the mean effective temperature on the stellar surface $\bar{T}_{\text {eff }}$,

- the gravity-darkening coefficient $\beta$,

- the stellar distance $d$ (to convert from linear to angular sizes).

For Fig. 4 we define a reference using the stellar model similar to Achernar (exactly as in our Paper I): $R_{\mathrm{eq}}=11 R_{\odot}, d=50 \mathrm{pc}$, $V_{\text {eq }} \sin i=250 \mathrm{~km} \mathrm{~s}^{-1}, i=60^{\circ}, M=6.1 M_{\odot}, \bar{T}_{\mathrm{eff}}=15000 \mathrm{~K}$, $\mathrm{PA}_{\text {rot }}=0^{\circ}$ (north direction), $\beta=0.25$ (theoretical value for radiative stellar envelopes; von Zeipel 1924), no differential rotation, with gravity darkening and limb darkening effects and Kurucz/Synspec line profile. These parameters correspond to $V_{\text {eq }}$ equal to $90 \%$ of the critical velocity $V_{\text {crit }}, R_{\mathrm{eq}} / R_{\mathrm{p}}=1.4$ and an equatorial angular diameter $\emptyset_{\mathrm{eq}}=2 R_{\mathrm{eq}} / d=2$ mas. To be compatible with the AMBER observations presented in Paper I, the simulations were performed around the hydrogen $\mathrm{Br}_{\gamma}$ line, $B_{\text {proj }}=75 \mathrm{~m}$ and $150 \mathrm{~m}, \mathrm{PA}=45^{\circ}$ and $90 \circ$, and $\lambda / \Delta \lambda=12000$.

Examples of maps of projected velocity and specific intensity in the continuum and in the line are shown in the top row of Fig. 4. The second row represents the 2D maps of the visibility modulus and visibility phase, photocenters (or centroid: the first-order term of the phase according to Mac Lauren development, Jankov et al. 2001), and normalized spectra deduced from the monochromatic intensity maps.

\section{Validation of SCIROCCO on VLTI/AMBER differential phases of Achernar}

To test and validate our semi-analytical code, we performed a $\chi^{2}$ minimization using the VLTI/AMBER differential phase data recorded on Achernar (cf. Sect. 2.1) and previously analyzed by Domiciano de Souza et al. (2012, Paper I).

The $\chi^{2}$ minimization was performed using a free Matlab implementation (from Matlab central file exchange) that we
Table 4. Limb-darkening parameters adopted, respectively, left to right, for Altair, $\delta$ Aquilae, and Fomalhaut.

\begin{tabular}{c|ccc}
\hline \hline \multirow{2}{*}{ Parameters } & Altair & $\delta$ Aquilae & Fomalhaut \\
\cline { 2 - 4 } & \multicolumn{3}{|c}{ Values } \\
\hline Turbulent velocity $V_{\mathrm{T}}$ & $2 \mathrm{~km} \mathrm{~s}^{-1}$ & $2 \mathrm{~km} \mathrm{~s}^{-1}$ & $2 \mathrm{~km} \mathrm{~s}^{-1}$ \\
\hline $\log g$ & $4 \mathrm{~cm} / \mathrm{s}^{2}$ & $3.5 \mathrm{~cm} / \mathrm{s}^{2}$ & $4.5 \mathrm{~cm} / \mathrm{s}^{2}$ \\
\hline Claret $T_{\text {eff }}$ & $\begin{array}{c}7250 \\
\text { to } \\
\text { Metallicity }\end{array}$ & $7250 \mathrm{~K}$ & $\begin{array}{c}8750 \\
\text { to } \\
9000 \mathrm{~K}\end{array}$ \\
\hline Spectral filter & -0.2 & 0 & 0 \\
\hline & $K$ & $K$ & $K$ \\
\hline
\end{tabular}

adapted for our needs using the generalized nonlinear nonanalytic chi-square fitting code, developed by $\mathrm{N}$. Brahms ${ }^{2}$. This code performs a fit to the measurements with known errors, and can use several Matlab library toolbox, such as the LevenbergMarquardt (LM) algorithm for faster convergence. We used the Monte Carlo method to confirm that the parameter uncertainties are normally distributed (Bevington \& Robinson 2002) in order to fit the simulated differential phases to the observations and to constraint the free parameters and their uncertainties. The free parameters are $R_{\text {eq }}, V_{\text {eq }}, i$, and $\mathrm{PA}_{\text {rot }}$. For our four stars cited in the introduction (Sect. 1), the VLTI/AMBER $\phi_{\text {diff }}$ observations were analyzed with the numerical model SCIROCCO presented in Sect. 3.

The limb-darkening parameters are fixed assuming the Claret function (Claret 2000a), as cited in the Sect. 3 and

2 http://www . mathworks . com/matlabcentral/fileexchange/ 9592-generalized-nonlinear-non-analytic-chi-squarefitting 
A\&A 569, A45 (2014)

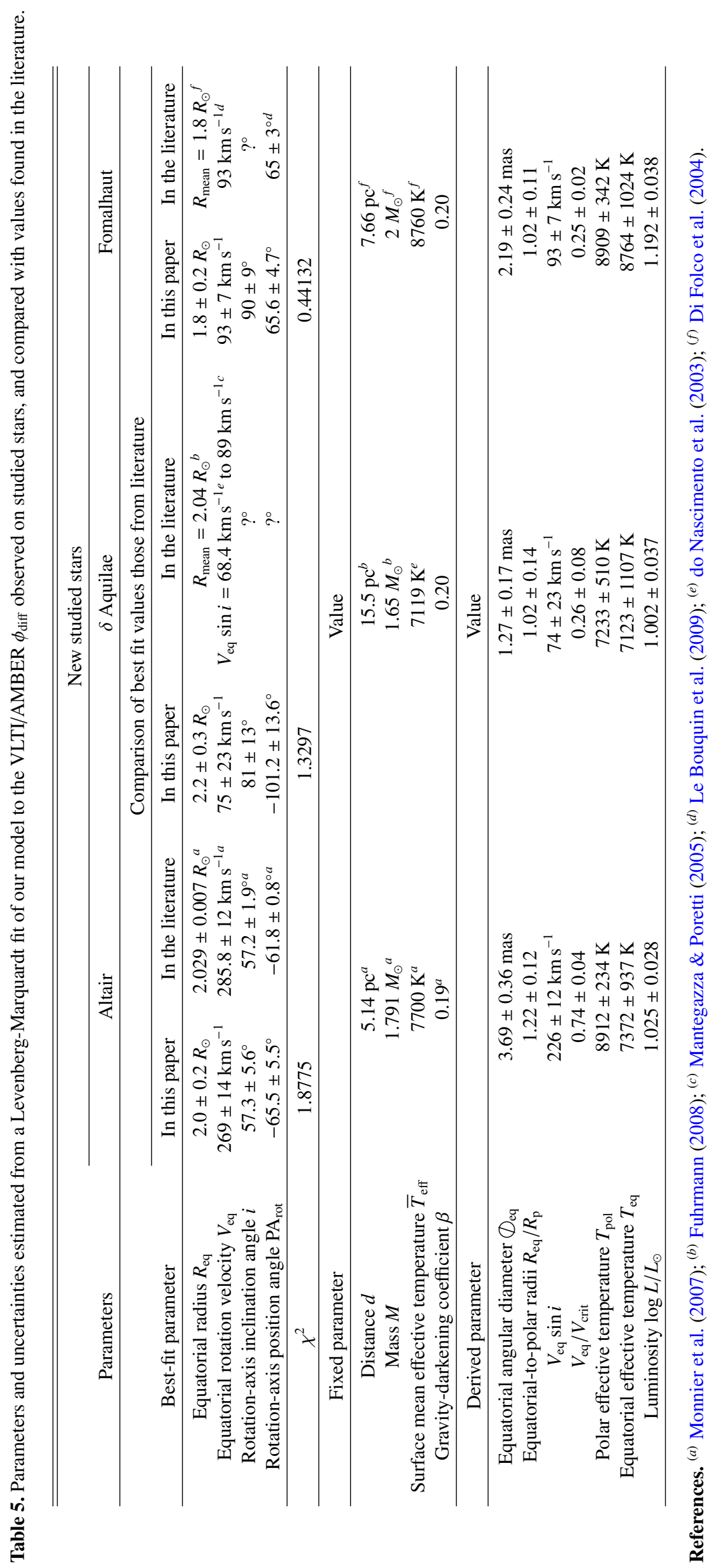



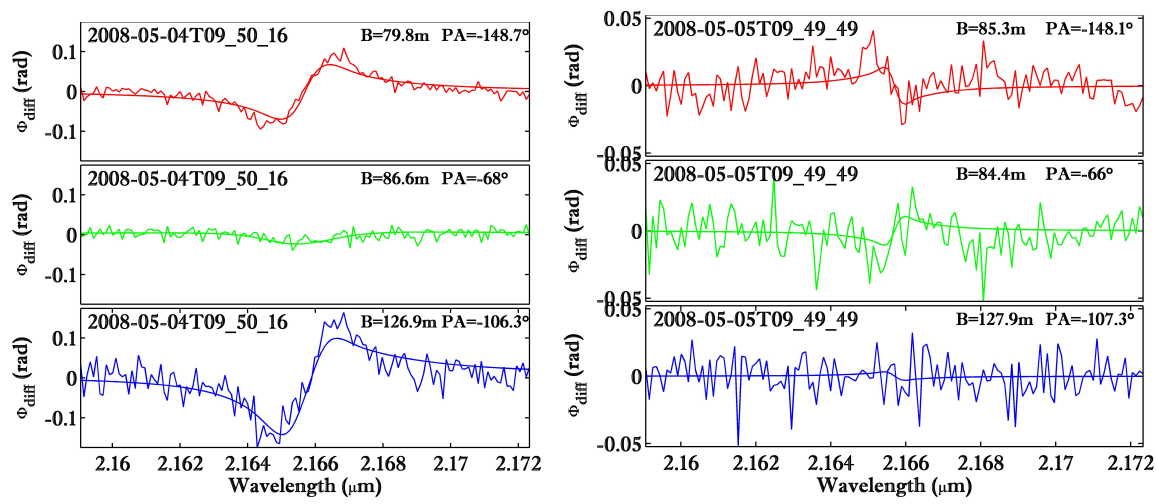

Fig. 6. The 6 VLTI/AMBER $\phi_{\text {diff }}$ measured on Altair and $\delta$ Aquilae around Br $\gamma$ at 2 different observing times (format YYYY-MM-DDTHH MM SS) and, for each time, three different projected baselines and baseline position angles, as indicated in the plots. The smooth curves superposed on the observations are the best-fit $\phi_{\text {diff }}$ obtained without a differential rotation.

summarized in the Table 2 . The gravity darkening coefficient $\beta$ has been fixed using the predicted theoretical relation between $\beta$ and temperature from Claret (2000b, 2012), as well as recent observational results. Based on the similar $\beta$ value found for studied rapid rotators (including $\beta$ Cas, which has spectral type F2III-IV, close to $\delta$ Aql), Che et al. (2011) recommend adopting a new standard $\beta=0.19$ for future modeling of rapidly rotating stars with radiative envelopes. However, here (as well as in Paper I) we use the value $\beta=0.2$, since this number of significant digits is consistent with our results, showing that $\phi_{\text {diff }}$ is not very sensitive to $\beta$ and to the relative uncertainties $\sim 10 \%$ in the derived parameters. The model parameters that were held fixed, following Paper I, are shown in Table 3. Following Espinosa Lara \& Rieutord (2011), which established the relation between the gravity darkening coefficient and the flatness of the star (see Fig. 4 of this paper), we deduced $\beta=0.15$ for Achernar, with which we have obtained similar results with $\beta=0.2$.

Figure 5 shows the best-fit $\phi_{\text {diff, }}$, together with the corresponding observations of Achernar. The best-fit values of the free parameters found with the LM algorithm are given in Table 3. As shown in these table and figures, best-fit model parameters and differential phases agree with the results from Paper I, within the measured uncertainties.

\section{SCIROCCO fitting of Altair, $\delta$ Aquilae, and Fomalhaut}

The free parameters adopted for Altair, $\delta$ Aquilae, and Fomalhaut model-fitting are $R_{\text {eq }}, V_{\text {eq }}, i$, and $\mathrm{PA}_{\text {rot }}$. They were calculated from previous works cited in the Sect. 3. The limbdarkening parameters are fixed assuming the Claret function (Claret 2000a), as described in Sect. 3, and are summarized in Table 4. The remaining model parameters were held fixed, as shown in Table 5. Still following Espinosa Lara \& Rieutord (2011), we deduced $\beta=0.19$ for Altair (exactly what we and Monnier et al. 2007, used). For $\delta$ Aquilae, and Fomalhaut we computed $\beta$ around 0.24 , and for these values we also obtained similar results to $\beta=0.2$ for these two last stars.

The best-fit values of the free parameters found with the LM algorithm are given in Table 5 as well. Figures 6 and 7 show the best-fit $\phi_{\text {diff }}$, together with the corresponding observations of $\delta$ Aquilae, and Fomalhaut. The uncertainties of the parameters estimated by the LM algorithm are $\approx 1 \%$.

\section{Conclusions and discussion}

In this work we applied the super-resolution capabilities provided by differential interferometry to measuring size (equatorial radius and angular diameter), projected velocity, and position angle of rotation axis of four fast-rotating stars: Achernar, Altair, $\delta$ Aquilae, and Fomalhaut. Our analysis was based on spectro-interferometric differential phases recorded with the VLTI/AMBER beam combiner, operating in its high and medium spectral resolution modes and centered on the $\mathrm{Br} \gamma$ line. All targets are only partially resolved by the interferometer in terms of fringe contrast (angular sizes $\sim 2-5$ lower than the theoretical angular resolution $\left.\lambda / B_{\max }\right)$.

The stellar parameters of the targets were constrained using a semi-analytical model to interpret the observations: SCIROCCO. This simplified algorithm dedicated to fast rotators was validated by showing that it leads to results in agreement with previous values obtained from an independent analysis of the same differential phase observations of Achernar (Paper I).

The observations of Altair, $\delta$ Aquilae, and Fomalhaut were fitted with SCIROCCO to determine their $R_{\text {eq }}$ (and $\varnothing_{\text {eq }}$ ), $V_{\text {eq }} \sin i$, and $\mathrm{PA}_{\text {rot }}$ from the VLTI/AMBER differential phases alone. These parameters are in good agreement (within the uncertainties) with values found in the literature and derived from different observables, such as spectra, squared visibilities, and differential phases.

In this work, we were able to deduce the rotation-axis inclination angle $i$ for $\delta$ Aquilae, and Fomalhaut $\left(i=81 \pm 13^{\circ}\right.$ and $90 \pm 9^{\circ}$, respectively) and the rotation-axis position angle $\mathrm{PA}_{\text {rot }}=-101.2 \pm 14^{\circ}$ for $\delta$ Aquilae, information that we have not found in the literature.

This work demonstrates that we can use the methodology shown in our Paper I for large specimens of fast rotators of different spectral types, and for several spectral interferometric observations.

The results from this work thus open new perspectives for using spectro-interferometry to study partially resolved fastrotating stars with present interferometers in order to simultaneously measure their sizes, projected velocities, and position angles.

Acknowledgements. This research made use of the SIMBAD database, operated at the CDS, Strasbourg, France, and of the NASA Astrophysics Data System Abstract Service. The author, M. Hadjara, acknowledges the support from the CRAAG Institue (Algeria), the Lagrange and OCA financial grants to carry on the present work, as well as the grants from the Fizeau European interferometry initiative (I2E). This research made use of the Jean-Marie Mariotti 
A\&A 569, A45 (2014)
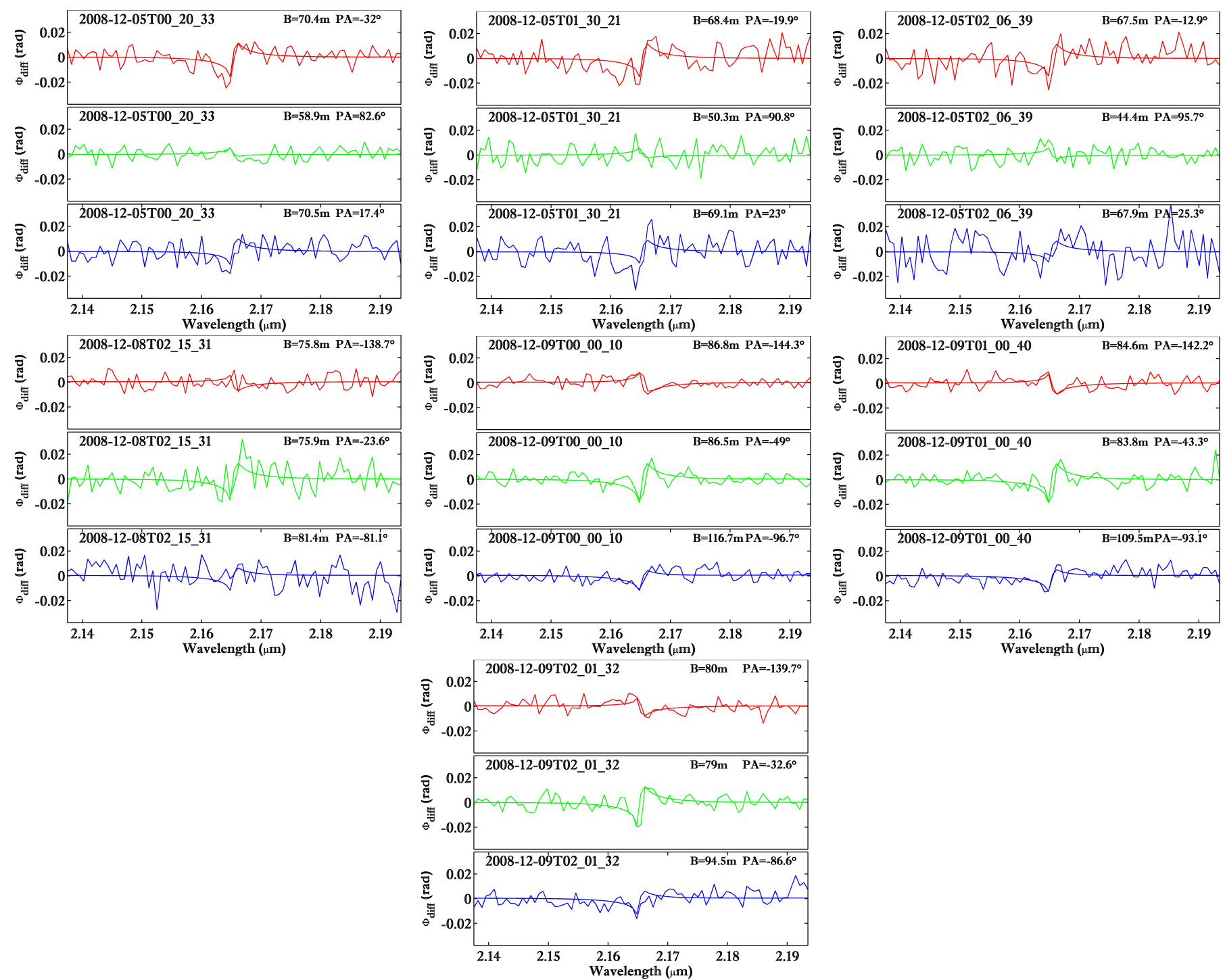

Fig. 7. The 18 VLTI/AMBER $\phi_{\text {diff }}$ measured on Fomalhaut around Br $\gamma$ at 7 different observing times (format YYYY-MM-DDTHH MM SS) and, for each time, three different projected baselines and baseline position angles, as indicated in the plots. The smooth curves superposed to the observations are the best-fit $\phi_{\text {diff }}$ obtained without a differential rotation (Domiciano de Souza et al. 2003), gravity-darkened Roche model, as described in Sect. 3. All the observed $\phi_{\text {diff }}$ points are shown here, knowing that the fit has been performed using all the wavelength points.

Center SearchCal service ${ }^{3}$ codeveloped by Lagrange and IPAG, and of the CDS Astronomical Databases SIMBAD and VIZIER ${ }^{4}$. This research made use of the AMBER data reduction package of the Jean-Marie Mariotti Center ${ }^{5}$. Special thanks go to Romain Petrov for his precious advice.

\section{References}

Abt, H. A., \& Morrell, N. I. 1995, ApJS, 99, 135

Aufdenberg, J. P., Mérand, A., Coudé du Foresto, V., et al. 2006, ApJ, 645, 664 Bevington, P. R., \& Robinson, D. K. 2002, Data reduction and error analysis for the physical sciences, 3rd edn. (McGraw-Hill)

Buzasi, D. L., Bruntt, H., Bedding, T. R., et al. 2005, ApJ, 619, 1072

Carciofi, A. C., Domiciano de Souza, A., Magalhães, A. M., Bjorkman, J. E., \& Vakili, F. 2008, ApJ, 676, L41

Che, X., Monnier, J. D., Zhao, M., et al. 2011, ApJ, 732, 68

Chelli, A., Utrera, O. H., \& Duvert, G. 2009, A\&A, 502, 705

Claret, A. 2000a, A\&A, 363, 1081

Claret, A. 2000b, A\&A, 359, 289

Claret, A. 2012, A\&A, 538, A3

\footnotetext{
3 Available at http://www . jmmc.fr/searchcal

4 Available at http://cdsweb.u-strasbg.fr/

5 Available at http://www.jmmc. fr/amberdrs
}

Collins, II, G. W., \& Sonneborn, G. H. 1977, ApJS, 34, 41

Cowley, A., \& Fraquelli, D. 1974, PASP, 86, 70

Delaa, O., Zorec, J., Domiciano de Souza, A., et al. 2013, A\&A, 555, A100

Di Folco, E., Thévenin, F., Kervella, P., et al. 2004, A\&A, 426, 601

do Nascimento, Jr., J. D., Canto Martins, B. L., Melo, C. H. F., Porto de Mello, G., \& De Medeiros, J. R. 2003, A\&A, 405, 723

Domiciano de Souza, A., Vakili, F., Jankov, S., Janot-Pacheco, E., \& Abe, L. 2002, A\&A, 393, 345

Domiciano de Souza, A., Kervella, P., Jankov, S., et al. 2003, A\&A, 407, L47

Domiciano de Souza, A., Kervella, P., Jankov, S., et al. 2005, A\&A, 442, 567

Domiciano de Souza, A., Hadjara, M., Vakili, F., et al. 2012, A\&A, 545, A130

Espinosa Lara, F., \& Rieutord, M. 2011, A\&A, 533, A43

Fuhrmann, K. 2008, MNRAS, 384, 173

Hadjara, M., Vakili, F., Domiciano de Souza, A., Millour, F., \& Bendjoya, P. 2012, in SF2A-2012: Proc. Annual meeting of the French Society of Astronomy and Astrophysics, eds. S. Boissier, P. de Laverny, N. Nardetto, et al., 533

Hadjara, M., Vakili, F., Domiciano de Souza, A., et al. 2013, in EAS Pub. Ser. 59, eds. D. Mary, C. Theys, \& C. Aime, 131

Holland, W. S., Greaves, J. S., Dent, W. R. F., et al. 2003, ApJ, 582, 1141

Hubeny, I., \& Lanz, T. 1995, ApJ, 439, 875

Jankov, S., Vakili, F., Domiciano de Souza, Jr., A., \& Janot-Pacheco, E. 2001, A\&A, 377, 721

Johnson, H. L., \& Morgan, W. W. 1953, ApJ, 117, 313 
M. Hadjara et al.: Beyond the diffraction limit of optical/IR interferometers. II.

Kalas, P., Graham, J. R., \& Clampin, M. 2005, Nature, 435, 1067

Kalas, P., Graham, J. R., Chiang, E., et al. 2008, Science, 322, 1345

Kanaan, S., Meilland, A., Stee, P., et al. 2008, A\&A, 486, 785

Kervella, P., \& Domiciano de Souza, A. 2006, A\&A, 453, 1059

Kervella, P., Domiciano de Souza, A., \& Bendjoya, P. 2008, A\&A, 484, L13

Kurucz, R. L. 1970, SAO Special Report, 309

Lachaume, R. 2003, A\&A, 400, 795

Lagarde, S. 1994, Ph.D. Thesis, Université de Sophia Antipolis, France

Le Bouquin, J.-B., Absil, O., Benisty, M., et al. 2009, A\&A, 498, L41

Maeder, A., \& Peytremann, E. 1972, A\&A, 21, 279

Mantegazza, L., \& Poretti, E. 2005, Commun. Asteroseismol., 146, 37

McAlister, H. A., ten Brummelaar, T. A., Gies, D. R., et al. 2005, ApJ, 628, 439

Meynet, G. 2009, in The Rotation of Sun and Stars, Lect. Notes Phys., 765 (Berlin: Springer Verlag), 139

Millour, F., Vannier, M., Petrov, R. G., et al. 2006, in EAS Pub. Ser. 22, eds. M. Carbillet, A. Ferrari, \& C. Aime, 379

Millour, F., Petrov, R. G., Chesneau, O., et al. 2007, A\&A, 464, 107

Millour, F., Valat, B., Petrov, R. G., \& Vannier, M. 2008, in SPIE Conf. Ser., 7013

Millour, F., Meilland, A., Chesneau, O., et al. 2011, A\&A, 526, A107

Monnier, J. D., Zhao, M., Pedretti, E., et al. 2007, Science, 317, 342
Peterson, D. M., Hummel, C. A., Pauls, T. A., et al. 2006, Nature, 440, 896 Petrov, R. G. 1989, in NATO ASIC Proc. 274: Diffraction-Limited Imaging with Very Large Telescopes, eds. D. M. Alloin \& J.-M. Mariotti (Dordrecht: Kluwer), 249

Petrov, R. G., Malbet, F., Weigelt, G., et al. 2007, A\&A, 464,

Reiners, A. 2006, A\&A, 446, 267

Reiners, A., \& Royer, F. 2004a, A\&A, 428, 199

Reiners, A., \& Royer, F. 2004b, A\&A, 415, 325

Royer, F., Grenier, S., Baylac, M.-O., Gómez, A. E., \& Zorec, J. 2002, A\&A 393, 897

Royer, F., Zorec, J., \& Gómez, A. E. 2007, A\&A, 463, 671

Slettebak, A. 1955, ApJ, 121, 653

Slettebak, A. 1982, ApJS, 50, 55

Stapelfeldt, K. R., Holmes, E. K., Chen, C., et al. 2004, ApJS, 154, 458

Tatulli, E., Millour, F., Chelli, A., et al. 2007, A\&A, 464, 29

Vakili, F., Mourard, D., Stee, P., et al. 1998, A\&A, 335, 261

van Belle, G. T. 2012, A\&ARv, 20, 51

van Belle, G. T., Ciardi, D. R., Thompson, R. R., Akeson, R. L., \& Lada, E. A. 2001, ApJ, 559, 1155

von Zeipel, H. 1924, MNRAS, 84, 665

Zhao, M., Monnier, J. D., Pedretti, E., et al. 2009, ApJ, 701, 209 
A\&A 569, A45 (2014)
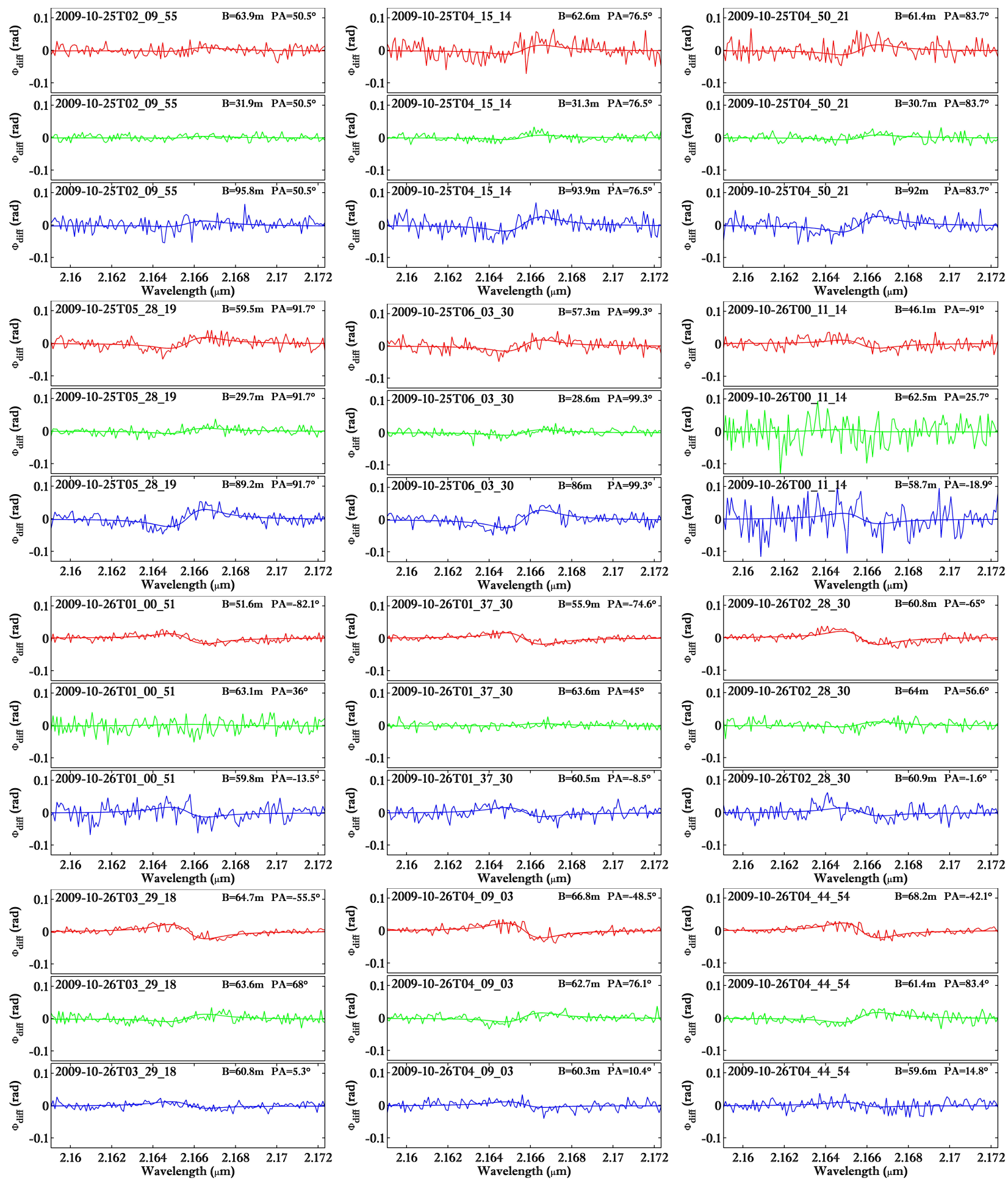

Fig. 5. The 84 VLTI/AMBER $\phi_{\text {diff }}$ measured on Achernar around $\operatorname{Br} \gamma$ at 28 different observing times (format YYYY-MM-DDTHH MM SS) and, for each time, three different projected baselines and baseline position angles, as indicated in the plots. The smooth curves superposed to the observations are the best-fit $\phi_{\text {diff }}$ obtained without a differential rotation (Domiciano de Souza et al. 2003), gravity-darkened Roche model, as described in Sect. 3. All the observed $\phi_{\text {diff }}$ points are shown here, knowing that the fit has been performed using all the wavelength points. 
M. Hadjara et al.: Beyond the diffraction limit of optical/IR interferometers. II.
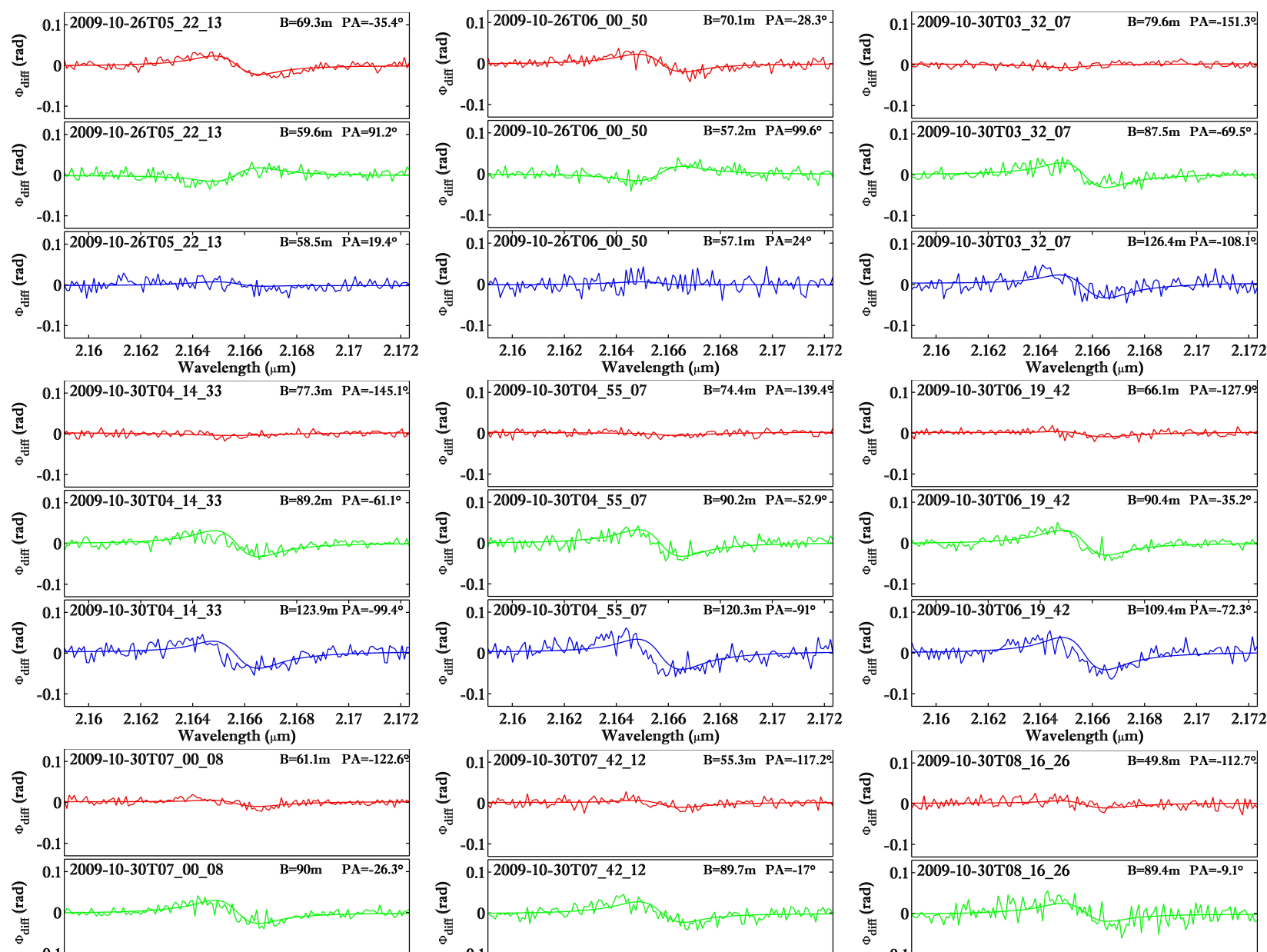

$-0.1$
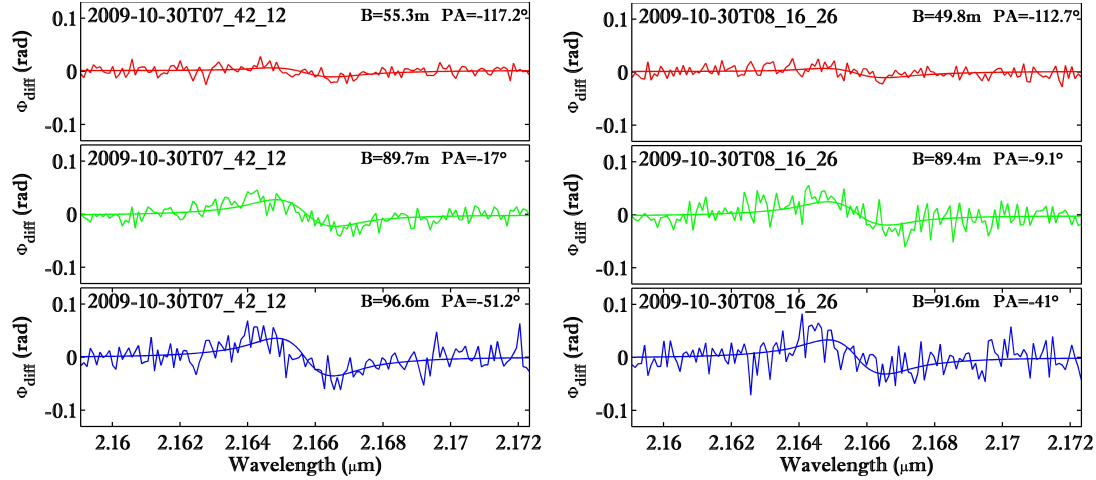

Wavelength $(\mu \mathrm{m})$

俤 0.1 2009-10-30T08_55_51 B=43m PA=-107.5

$-0.1$

0.1-2009-10-30T08_55_51 $\mathrm{B}=89.3 \mathrm{~m} \quad \mathrm{PA}=0.1^{\circ}$

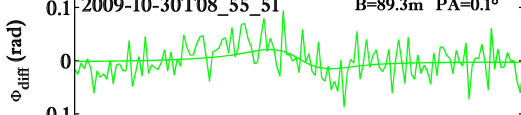

2.16 $2.162 \quad 2.164 \quad 2.166 \quad 2.168$ 2.17
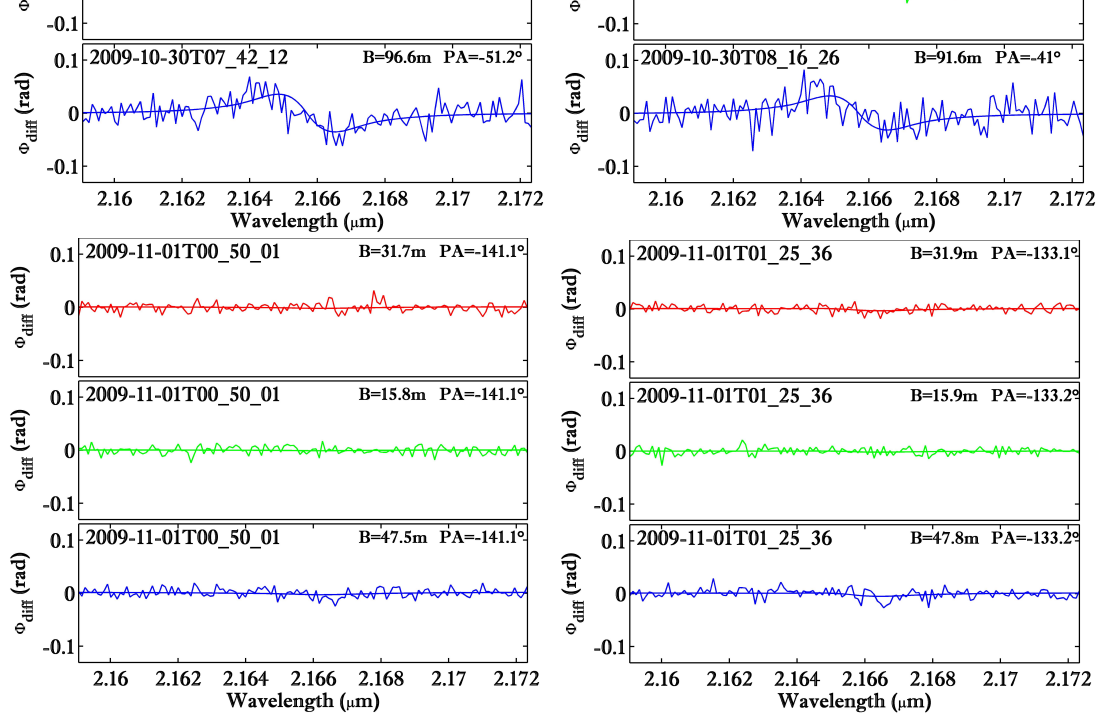

Fig. 5. continued. 
A\&A 569, A45 (2014)
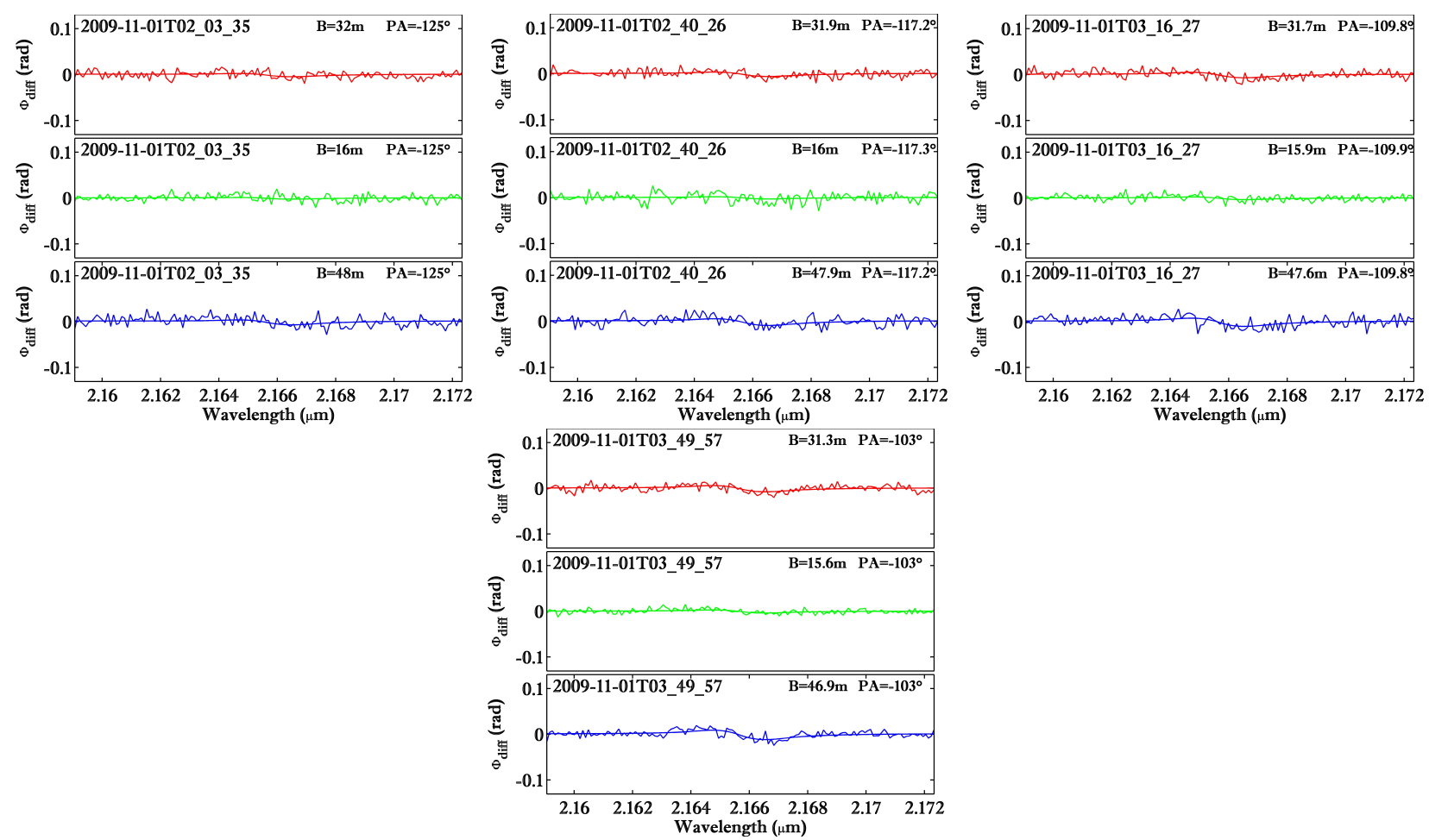

Fig. 5. continued. 